\title{
Rehabilitation Following Anatomic Total Shoulder Replacement for Osteoarthritis
}

James Blacknall MSC BSC (Hons)

Advanced practice shoulder and elbow physiotherapist, Trauma and Orthopaedics Department, Sherwood Forest Hospitals NHS Foundation Trust, Nottinghamshire, United Kingdom

Amit Sharad Bidwai MBCHB, MRCSEd, FRCS (Tr \& Orth)

Consultant trauma and orthopaedic surgeon, Trauma and Orthopaedics Department, Sherwood Forest Hospitals NHS Foundation Trust, Nottinghamshire, United Kingdom

\section{ABSTRACT}

Advances in anatomic total shoulder replacement (TSR) have seen this become an established surgical intervention for patients suffering from glenohumeral osteoarthritis (OA). A growing evidence-base stresses good prosthesis survivorship, low complication rates, and reproducible improvements to patients' quality of life and function. Despite these advances, the rehabilitation of patients undergoing anatomic TSR has received relatively little attention. This clinical commentary discusses a specific clinical method taken to manage patients undergoing anatomic TSR for glenohumeral OA and an intact rotator cuff. It outlines the evaluation-based rehabilitation approach developed between surgeons and physiotherapists at the Sherwood Forest Hospitals NHS Foundation Trust in Nottinghamshire, United Kingdom. It is hoped this commentary will generate further interest in this area and help drive advances in the outcomes and rehabilitation of patients undergoing TSR.

Blacknall, J., \& Bidwai, A. S. (2020). Rehabilitation following anatomic total shoulder replacement for osteoarthritis. New Zealand Journal of Physiotherapy, 48(2), 80-91. https://doi.org/10.15619/NZJP/48.2.04

Key Words: Rehabilitation, Physiotherapy, Total Shoulder Replacement

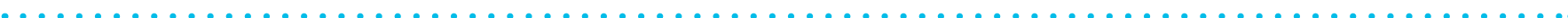

\section{INTRODUCTION}

Anatomic total shoulder replacement (TSR) has become an established and popular treatment choice for the patient with osteoarthritis (OA) of the shoulder (Denard \& Ladermann, 2016; Mueller \& Hoy, 2014). As anatomic TSR design and understanding have developed, a growing evidence base has helped inform patient outcomes (Denard \& Ladermann, 2016; Razmjou et al., 2014). The risks and benefits of anatomic TSR surgery are increasingly understood and reproducible (Bohsali et al., 2017; Young et al., 2011), with excellent long-term prosthesis survivorship, and improved quality of life and functional independence for patients (Singh et al., 2011; Styron et al., 2015; Werner et al., 2017). However, little attention has been given to the optimal rehabilitation of patients undergoing anatomic TSR, despite a consensus that rehabilitation plays an important role in optimising the outcomes for such patients (Bullock et al., 2019).

This clinical commentary, describing a rehabilitation approach, has been developed based on our experiences and the available science to meet the challenge of striving to optimally manage the patient undergoing anatomic TSR for the management of shoulder OA with an intact rotator cuff. It aims to provide a timely update on anatomic TSR rehabilitation, and describe the principles behind our approach and how these can address some of the inconsistencies noted in TSR rehabilitation guidelines (Bullock et al., 2019). Specifically, we aim to show how we developed our approach to meet the surgical implications of subscapularis exposure, the timing and rationale for rehabilitation progressions, and how the growing understanding of psychosocial factors that influence patients may be considered to ameliorate patient outcomes.

As stated by Bullock et al. (2019), the indications and pathoanatomy of patients undergoing anatomic or reverse TSR are different, making apposite rehabilitation essential. We feel it is imperative to understand the difference between rehabilitation of the anatomic and reverse TSR, making this commentary distinct from guidelines previously presented for reverse TSR (Blacknall \& Neumann, 2011).

\section{PRE-OPERATIVE STAGE}

There is increasing awareness that patient expectation and experience can significantly impact clinical outcome. For instance, surgical and recovery expectations influence health outcomes, such as quality of life and function (Henn et al., 2011). Furthermore, patients' preoperative expectations of orthopaedic surgery have been shown to vary by diagnosis, sex, education, level of function, and general health status (Henn et al., 2011). Accordingly, the pre-operative clinic provides an ideal opportunity to manage and discuss these aspects whilst also providing the forum to convey to the patient some important aspects of their rehabilitation.

In the pre-operative clinic, we discuss with the patient and demonstrate what their recovery and rehabilitation following surgery will involve in terms of exercise, sling utilisation, recommended sleep positions, and functional dos and don'ts. This allows the patient to practice functional tasks prior to surgery, such as negotiating stairs or using transport, thereby facilitating an understanding of how they will manage in the immediate post-operative phase. 
There is a growing body of literature that has identified patient psychological factors and the influence these have on treatment outcomes for patients with chronic shoulder pain (Chester et al., 2018; Gil et al., 2018). Whilst it is beyond the scope of this review to explore these wide-reaching themes in detail, the literature related to shoulder arthroplasty does provide us with some valuable insights that we can incorporate into our rehabilitation strategies (Tokish et al., 2017).

Depression and anxiety (which are treatable conditions), resilience, defined as "the ability to recover from a stressful event" (Tokish et al., 2017, p. 753), and self-efficacy, which refers to "one's belief in one's ability to succeed in specific situations" (Bandura, 1977), have varying effects on outcomes following TSR. Therefore, until further research enlightens the causality between these disorders and the outcome of TSR, caution is required when predicting recovery (Cho et al., 2017; Styron et al., 2015; Werner et al., 2017). However, if we feel these psychological factors are likely to be a barrier to recovery, we pursue medical input with the patient, usually through their general practitioner, where appropriate treatment options can be discussed. A supportive, coaching, and holistic role in the pre-operative clinic with patients and throughout rehabilitation helps to positively influence such factors, as demonstrated by Picha and Howell (2018).

At this stage, we take time to explain to patients how to use problem-solving to manage activities of daily living following surgery to support goal setting, and to outline the benefits of exercise during rehabilitation. We feel that with an empathetic approach, this provides a foundation to empower patients, instil confidence, and improve resilience and self-efficacy. Written information is also given to patients to support this education process and provide a resource that they can refer to during rehabilitation and reflect on with family members and/or friends.

Social support is important for patients, and we are keen to encourage and engage with any family/friends that the patient may want included in the pre-operative clinic and, indeed, throughout rehabilitation. Involving family can help support patients who may feel a sense of helplessness and anxiety about coping following surgery (Picha \& Howell, 2018).

\section{THE OPERATION: SURGICAL APPROACH AND INSIGHTS}

Irrespective of the chosen anatomic TSR prosthesis, there are some fundamental surgical principles that helped inform our rehabilitation approach.

\section{Surgical approach}

Typically, anatomic TSR is performed through the deltopectoral interval (Mueller \& Hoy, 2014; Wolff \& Rosenzweig, 2017). Through this fascial split the surgeon gains access to the shoulder joint either via a subscapularis division (peel technique or mid-substance tenotomy) or a lesser tuberosity osteotomy (LTO) approach (Armstrong et al., 2016; Choate et al., 2018).

Numerous biomechanical studies have examined the commonly used subscapularis peel (SP), subscapularis tenotomy (ST) or LTO techniques. It is controversial as to which of these techniques is the most advantageous. Of importance, however, is subscapularis function following anatomic TSR. Subscapularis dysfunction is associated with an inferior clinical result, evidenced by pain, weakness or anterior instability (Armstrong et al., 2016; Choate et al., 2018). A recent meta-analysis of the biomechanical data reported the load-to-failure of the initial repair to be stronger for the LTO approach, while there was no statistically significant difference under cyclic load testing between the different SP and ST techniques (Schrock et al., 2016). Clinical results also trend toward supporting the LTO approach, where a recent systematic review found subscapularis healing and integrity appeared to favour the LTO technique, with the rate of intact tendon after surgery for LTO (93.1\%) being significantly better than that of the ST $(75.7 \%)$ or SP (84.1\%) technique (Choate et al., 2018).

Rehabilitation, therefore, needs to balance the considerations for optimal tissue healing of the subscapularis repair constructs whilst avoiding the effects of deleterious disuse. The lack of specificity regarding subscapularis management in postoperative rehabilitation following anatomic TSR has recently been highlighted (Bullock et al., 2019).

\section{Soft tissue balance}

Soft tissue balancing (the close interplay between the capsular and tendon soft tissue envelope, the bony architecture of the humerus and glenoid, and implant positioning) impacts significantly on postoperative rehabilitation (Mueller \& Hoy, 2014; Stephens et al., 2017). Firstly, during surgery, care is taken to release the soft tissue envelope that is often contracted due to $\mathrm{OA}$ to ensure an adequate capsular laxity that is required for normal shoulder motion. A long head of biceps tenodesis is often performed, allowing improved external rotation range while not causing any obvious functional loss (Mueller \& Hoy, 2014).

Secondly, glenohumeral OA produces consistent bony changes, although the severity will depend upon the disease progression (Malhas et al., 2016; Matsen et al., 2004). Osteophytes must be resected adequately to avoid unwanted motion loss and any glenoid wear, classically posterior. These should be effectively dealt with to achieve normal joint stability and avoid an abnormal length-tension relationship between the subscapularis and infraspinatus (Malhas et al., 2016; Mueller \& Hoy, 2014).

Thirdly, the correct implant positioning and placement will ensure the arthroplasty is not "overstuffed" or predisposed to instability, thereby preserving the requisite shoulder motion (Mueller \& Hoy, 2014; Stephens et al., 2017).

Information regarding soft tissue balance and post-operative range of movement parameters is invaluable if we are to optimise the patient's functional outcome. Fortunately, there are some helpful approaches that can be used to help advise us in this regard (Matsen et al., 2004).

\section{Testing on the table}

The 40/50/60 guideline is a popular method of detailing the soft tissue range of movement following TSR (Matsen et al., 2004). According to this guideline, 40 is the degree of external rotation with the arm at the side following soft tissue approximation, 50 is the percentage of translation in relation to the glenoid width on the posterior drawer test while 60 is the degree of internal rotation with the arm in abduction (Matsen et al., 2004). 
Information on subscapularis biomechanics is particularly important, given the poor outcomes associated with its failure (Armstrong et al., 2016; Choate et al., 2018). An understanding of the range of external rotation that is safely available following surgery will inform our exercise prescription (Wolff \& Rosenzweig, 2017). While assessing soft tissue balance following anatomic TSR can be a diverse process, it is important that this guidance is communicated to the rehabilitation team by the surgeon(s) to facilitate an optimal and safe postoperative recovery.

\section{Complications and survivorship}

Complication rates following anatomic TSR are low, with a large review finding rates for instability of $1 \%$ with rotator cuff tear, postoperative fracture, neural injury, and infection all below 1\% (Bohsali et al., 2017). Instability typically presents as either anterosuperior escape associated with poor subscapularis function, or as posteroinferior subluxation (Matsen et al., 2004). We should be wary of anterosuperior instability in patients with pain, unexpected poor flexion, and observable or palpable increased anterior translation of the humeral head at rest or during early flexion, particularly if there are any concerns with the integrity of subscapularis. Posteroinferior instability often presents as pain and an observable or palpable posterior translation and "clunk" during flexion movements.

Superior rotator cuff tear (not involving the subscapularis) following anatomic TSR is, again, rare, but a clinical suspicion should be raised in patients who have increasing pain, unexpected loss of movement, and weakness on rotator cuff testing. There is some thought that an "overstuffed" prothesis may increase this risk as the oversized humeral head places more tension and stress on the in-situ rotator cuff tendons (Matsen et al., 2004). Good understanding of these issues will facilitate early identification during rehabilitation and should prompt physiotherapists to discuss these with the surgical team before continuing rehabilitation.

Patients are naturally keen to understand how long their shoulder replacement will last. Singh et al. (2011) reported TSR implant survivorship rates for OA of $95 \%$ at 5 years, $91 \%$ at 10 years, and $81 \%$ at 20 years. Young et al. (2011) reported survivorship rates for patients undergoing TSR for OA of $99.1 \%$ at 5 years, $94.5 \%$ at 10 years, and $79.4 \%$ at 15 years, with glenoid component revision taken as the end point. Survivorship rates with radiological loosening taken as the end point revealed $99.1 \%$ at 5 years, $80.3 \%$ at 10 years and $33.6 \%$ at 15 years.

It is reassuring that implant loosening or migration is rare during the rehabilitation period. However any sudden onset of pain, particularly where associated with loss of movement and crepitus or grating, should prompt immediate discussion with the surgical team. While these survivorships rates are promising and provide useful information for patients, there is some acknowledgment in the literature of risk factors for less favourable rates, namely patients with higher activity levels and who are younger at the time of surgery (Farng et al., 2011).

\section{REHABILITATION PATHWAY}

Our rehabilitation pathway is divided into four distinct elements: the pre-operative clinic, as discussed above, followed by the early postoperative phase "protected mobility", the intermediate postoperative phase "active recovery", and the late postoperative phase "functional reintegration".

Early postoperative phase: "Protected mobility"

The philosophy of the early rehabilitation phase is to manage the twin aims of protecting the shoulder tissues whilst avoiding the unwanted effects associated with surgical trauma, pain, and poor patient adherence to rehabilitation recommendations (Ahmad et al., 2015).

\section{"Protected mobility" education and functional advice} Patients are educated on how to avoid forces through the arm. For example, patients are shown how not to use the operated arm when sitting and rising from a chair or getting out of bed to prevent unnecessary stress risers through the arm and loading the subscapularis repair construct.

We find sleep position advice particularly useful. Maintaining the shoulder joint in a neutral position (shown in Figure 1) provides patients with practical steps on how to position themselves comfortably and confidently for sleep while also providing pain relief and, therefore, better quality rest (Wolff \& Rosenzweig, 2017) - both important for an optimal recovery (Ahmad et al., 2015). Advice on resting positions is developed to facilitate simple functional tasks, such as washing and dressing, with the sling removed, again helping to ensure patients do not unnecessarily load the arm (Gurney et al., 2016). Emphasising

\section{Figure 1}

\section{Sleeping Positions}
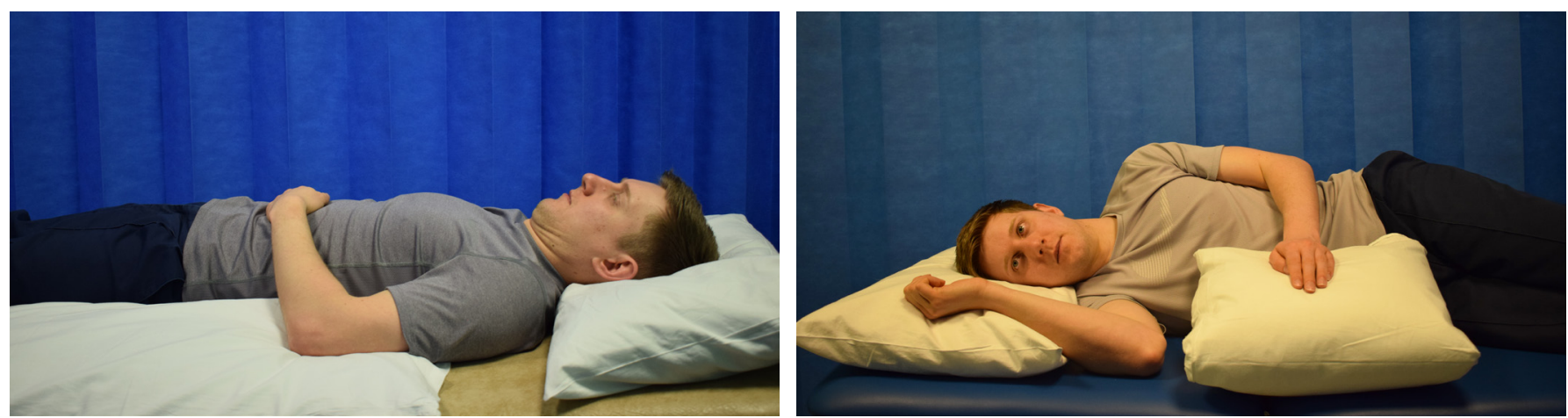

Note. Left panel: Supine sleep position. Right panel: Side-lying sleep position. 
the need to adopt these practical and functional methods in the early phase of rehabilitation minimises repetitive loads on the subscapularis repair that may lead to clinical failure (Choate et al., 2018; Schrock et al., 2016).

This coaching and supportive approach helps patients cope and manage well in the postoperative stages. Self-efficacy and rehabilitation adherence can be improved by empowering patients to perform tasks correctly, setting goals, positively reinforcing the information from the preoperative stage, discussing pain management and how to pace activities, and reducing any fear of failure the patient may harbour (Picha \& Howell, 2018).

Education around the need to manage a sling correctly is provided. This is particularly important in vulnerable environments (e.g. shopping/using transport) as high levels of subscapularis activity occur when putting on and taking off a sling (Gurney et al., 2016). Biomechanically the subscapularis repair constructs have been shown to have good load-tofailure strength (average $350 \mathrm{~N}$ ) and an ability to withstand displacement on cyclic loading (Schrock et al., 2016). Therefore, we feel patients do not need to be routinely immobilised postsurgery.

\section{"Protected mobility" exercise prescription}

The early phase of exercises aims to mobilise the shoulder joint, helping promote functional independence; and avoid potential stiffness, contracture, and pain management problems.

Exercises are implemented according to the communication of post-implantation soft tissue balance and any potential complications that the surgical team feel may have implications for rehabilitation, for example avoiding a certain range of external rotation if the subscapularis repair was unduly tensioned or vulnerable in such a position. Patients are taught to perform the exercises with the operated arm as relaxed as possible; in essence, we want the exercise to be as "passive" as possible, ensuring minimal forces across the healing tissues (Edwards et al., 2017; Jung et al., 2016). Anecdotally, patients are far more comfortable and successful with these exercises when they are relaxed than if they are tense and nervous when moving the arm. Again, this reflects the supportive, educative and holistic role physiotherapists inherently take with patients, providing positive feedback, and reassuring and coaching patients as they recover.

There is a reasonably linear relationship between muscle electromyography and force during near isometric and constant velocity contractions (Edwards et al., 2017; Thigpen et al., 2016). The early phase of these exercises show low muscle activity with electromyographic studies, and we are confident that we are not inducing forces through the healing tissues that have been found to cause failure in-vitro (Schrock et al., 2016).

\section{Flexion}

Pendulum, supine active-assisted flexion and pulley exercises all show low muscle activity (Edwards et al., 2017; Mazuquin et al., 2018), and in the acute post-operative setting are well tolerated by patients, and carry little risk of unduly loading and stressing the healing tissues (Mazuquin et al., 2018). Patients are encouraged to progress through a range of motion as comfort allows, and this range of motion is not routinely constrained.

\section{External rotation}

External rotation is an important movement to regain for normal shoulder motion, with $35^{\circ}$ being required for maximal elevation (Browne et al., 1990). Patients with poor external rotation range may be susceptible to subacromial pain syndrome, as the greater tuberosity cannot escape from underneath the acromial arch (Browne et al., 1990; Matsen et al., 2004), a phenomenon to factor into postoperative rehabilitation.

Supine external rotation using a stick with the arm in a supported neutral position shows low muscle activity (Thigpen et al., 2016). However, if injudiciously applied, this will stress the subscapularis repair construct (Edwards et al., 2017; Wolff \& Rosenzweig, 2017). Recognising the rehabilitation specificity of the subscapularis approach, careful use of supine external rotation based on the intra-operative soft tissue balance is recommended to ensure the patient does not push into overt pain. (Bullock et al., 2019).

\section{Extension}

A standing passive extension using a stick, initiated as patient comfort allows, is again well tolerated by patients with low muscle activity (Thigpen et al., 2016). Care is required to ensure this movement is comfortable.

\section{Exercise dose and technique}

We expect patients to perform their exercise programme twice daily to begin with and suggest 10 repetitions for each exercise. However, the repetitions and frequency of performance are modified depending on how the patient is progressing. For example, in the case of a patient whose range of movement is not where it should be, but who is comfortable with the exercise programme, we suggest increasing the range of movement and monitoring the patient.

Patients' exercise technique and understanding of the follow-up physiotherapy sessions are checked to ensure the exercises are being performed correctly and the functional advice described above is being followed (Ahmad et al., 2015). Again, we are alert to potential barriers or psychosocial factors that may be affecting recovery, as discussed previously (Tokish et al., 2017). For example, group rehabilitation may be appropriate to augment the patient's home programme if it is felt that the patient would benefit from the peer support in a group environment, either via their own or vicarious experience (Picha \& Howell, 2018).

Intermediate postoperative phase: "Active recovery" In this phase patients continue to follow their active-assisted exercise programme and joint protection advice from the protected mobility phase. Movement re-education and active exercises are introduced through an evaluation-based criterion, developing the active recovery process.

Evaluation-based criteria

The evaluation-based criteria are founded on:

1. Time since surgery.

2. Patient's tissue quality. 


\section{Surgical considerations.}

4. Patient's rehabilitation progress.

5. Clinical findings.

The first three criteria are evidenced from the operation note and/or surgical team, along with an understanding of any physiological factors that may alter healing and recovery in the patient, be that medical influences, such as diabetes, or lifestyle factors, such as smoking. Criteria 4. and 5. relate to the patient's subjective report of their symptoms and recovery, and objective information from physical testing. The evaluation-based criteria are invaluable for the safe and optimal progression of the patient's rehabilitation.

The biomechanical literature shows some consistency in the subscapularis repair mode of failure, with the majority of LTO failing at the bone interface, while soft tissue failure with suture cut through at the muscle/tendon is seen with tenotomy (SP, ST) repairs (Ahmad et al., 2015; Schrock et al., 2016). The various subscapularis techniques - bone-to-bone (LTO), tendon-to-bone (SP) and tendon-to-tendon (ST) - will have different modes of healing. Therefore, until adequate healing has occurred, there is the spectre of tendon failure, which should be factored into our rehabilitation (Choate et al., 2018; Wolff \& Rosenzweig, 2017).

The LTO that does not violate the tendon should theoretically heal quicker than a subscapularis repair, as bone healing takes less time and is more understood and predictable than tendon healing. This allows us to accelerate exercise progressions in these patients and ensures rehabilitation is specific to the subscapularis approach (Bullock et al., 2019).

TSR with an LTO (3-weeks post-surgery)

At three weeks post anatomic TSR, patients with an LTO are progressed if they meet our evaluation-based criteria: they have adequate tissue quality, there are no surgical factors that warrant a more conservative progression, they report minimal pain with their rehabilitation programme to date, and they are pain free on our two clinical progression tests. These two tests (Figures 2-3) are:

1. Supported active internal and external rotation with the elbow flexed to $90^{\circ}$ in supine.

2. Active short-lever $0-90^{\circ}$ shoulder flexion "forward punch" in supine.

During loaded external rotation, subscapularis demonstrates low levels of activity (Edwards et al., 2017; Thigpen et al., 2016).

The supine "forward punch" movement has also shown a low level of subscapularis muscle activity (Wattanaprakornkul et al., 2011), even when loaded, so we feel the unloaded short-lever technique described above is a rational active functional testing position.

\section{Figure 2}

Supported Active External to Internal Rotation, Performed in Supine
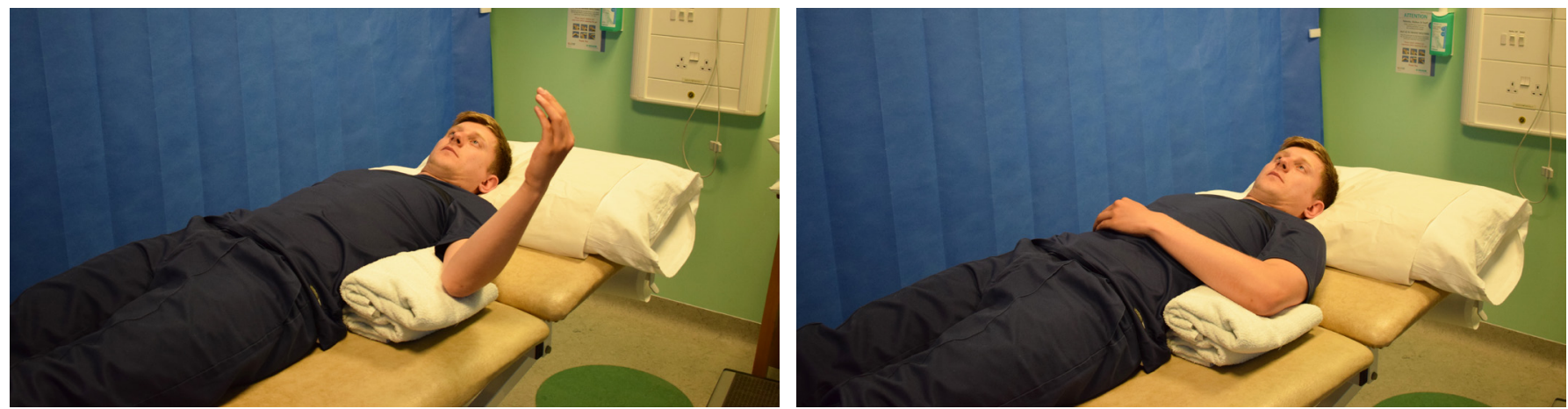

Note. Left panel: Starting position. Right panel: End position.

\section{Figure 3}

Active Short-Lever Flexion (0-90) "Forward Punch", Performed in Supine
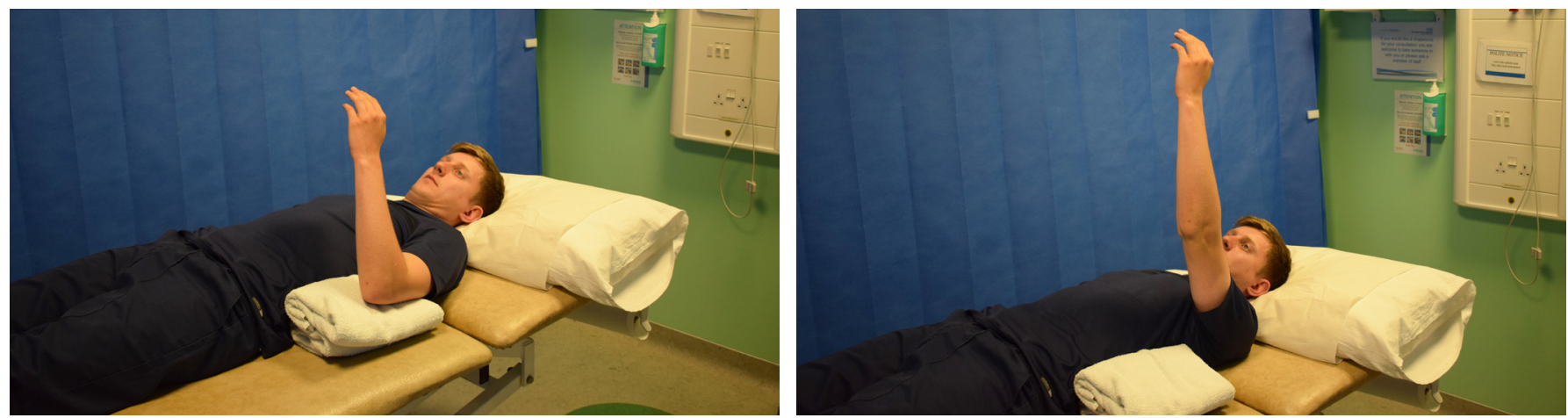

Note. Left panel: Starting position. Right panel: End position. 
Patients that satisfy these criteria are progressed to weaning off their sling, commence hand behind the back active-assisted exercise progressions, and can start active range of movement work with an emphasis on good quality motor control.

Not all patients will meet our criteria at this stage. This is usually due to either tissue quality or surgical considerations, or they report poor pain control with their exercises to date and fail our clinical progression tests (namely pain with the active external rotation and "forward punch" test). In this scenario, we are careful to ensure that patients have been following their rehabilitation plans and check for any potential barriers to recovery that may be affecting their progress.

Poor pain management may be one factor to discuss with patients at this stage. Patients can be reluctant to use their prescribed analgesics due to fears of masking pain or, indeed, they may be experiencing unwanted side effects, such as an upset stomach or constipation. Exploring these issues and problem-solving them with patients, and involving the medical team if necessary are important steps for optimising rehabilitation. Again, reinforcing a positive recovery expectation, coaching, and supporting patients with positive feedback, goal setting, and engaging the patient's social support network are all methods to help improve self-efficacy and rehabilitation adherence (Chester et al., 2018; Picha \& Howell, 2018).

Patients are reviewed at follow-up physiotherapy sessions until we feel they can progress. Ongoing pain and the inability to complete our clinical tests indicates the need to review the patient's progress with the surgical team.

TSR with an SP or ST (4-weeks post-surgery) Anatomic TSR patients with an SP or ST approach are evaluated at the four-week postoperative stage. Those fulfilling our evaluation-based criteria discussed above can start sling weaning and the hand-behind-the-back active-assisted exercise progressions, affording a little more functional independence and engendering our supportive holistic recovery approach. However, we do not start active exercise progressions and movement control until evaluated again at the six-week stage to help protect the subscapularis tendon repair (Mazaquin et al., 2018; Wattanaprakornkul et al., 2011). For the patients with an SP or ST approach who do not meet our criteria at this stage, we apply the strategies previously discussed for the LTO approach.

\section{"Active recovery" movement control exercises}

These progressions should be symptom free, and if they are not, patients are re-evaluated during physiotherapy sessions until we deem progression appropriate. Patients are encouraged to maintain the exercise frequency that has already been established, and then develop the movement control work as symptoms allow in a "little-and-often" routine. These exercises aim to achieve improved motor control where repetition and frequency is the goal, rather than a strength training/overload principle of exercise prescription. Such progressions help empower patients by reintegrating daily life activities, assisting to develop their resilience and self-efficacy, and facilitating adherence to rehabilitation (Picha \& Howell, 2018).

Active flexion and external rotation from supine are comfortable starting transitions. As symptoms allow, these can be progressed to sitting or standing active flexion and external rotation along with extension and internal rotation physiological range of movement exercises (Edwards et al., 2017; Thigpen et al., 2016).

Patients with shoulder OA often have altered movement patterns (Alta et al., 2014; de Toledo et al., 2012) due to the pain, stiffness, and loss of function caused by the disease process. These patterns represent compensatory movement strategies that following anatomic TSR should be re-educated during rehabilitation; we consider a normal movement pattern a prerequisite to achieving normal function. Therefore, as patients are prescribed the active range of movement exercises above, they are taught with an emphasis on movement dissociation, particularly glenohumeral joint from scapulothoracic joint to improve their kinesthetic and proprioceptive awareness, which have been shown to be altered in TSR patients (Alta et al., 2014; de Toledo et al., 2012). Mirrors and/or video feedback are useful methods to employ to help patients understand the movement faults we want to address. Patients are also encouraged to incorporate movement dissociation into their other activeassisted exercises and simple functional tasks to enhance the cortical carry-over and motor relearning process. Importantly, the patient must have adequate passive/active-assisted movement before they can use this range actively.

Late postoperative phase: "Functional reintegration" The next stage of rehabilitation aims to build upon the activeassisted and active-movement work already under way with the introduction of loading exercises to progress the strength, stamina, and efficiency of the shoulder complex to enhance functional reintegration.

\section{TSR with an LTO (6 weeks post-surgery)}

At 6 weeks, patients with an LTO are progressed according to our evaluation-based criteria. If there are no concerns with tissue quality or surgical considerations, and patients have experienced minimal pain with rehabilitation to date, clinical progression tests are applied. If patients are pain free with therapist-resisted supine internal rotation from neutral rotation, and can perform the "forward punch" test loaded in supine (Figure 4), loaded rehabilitation exercises are started. The clinical progression tests do not require any specific equipment. The internal rotation test utilises physiotherapist resistance to gentle isometric internal rotation in neutral to judge the quality of contraction and symptom reproduction (Figure 5). For the "forward punch" test, we start with a small weight, typically $0.5 \mathrm{~kg}$, and if the patient is symptom free on testing, we use this as the starting load for exercise progressions. For any patient that fails our functional tests (i.e. pain with resisted internal rotation and/or with the loaded forward punch) we check their exercise programme for any factors that may be affecting their progression. Patients are not progressed until re-evaluation at subsequent physiotherapy sessions, whereby the clinical progression tests are repeated.

We apply this evaluation-based approach because whilst the LTO has been shown to have an excellent healing rate, literature suggests it is not immune from complications (Choate et al., 2018; Denard \& Ladermann, 2016). A recent review identified a small number of tuberosity failures in relatively young (mean age 52 years) male patients with a muscular build, where the LTO failure occurred within 2 to 3 months following surgery, resulting from little or minor trauma (Shi et al., 2015). Thus, 
Figure 4

Loaded Short-Lever Flexion (0-90') "Forward Punch", Performed in Supine

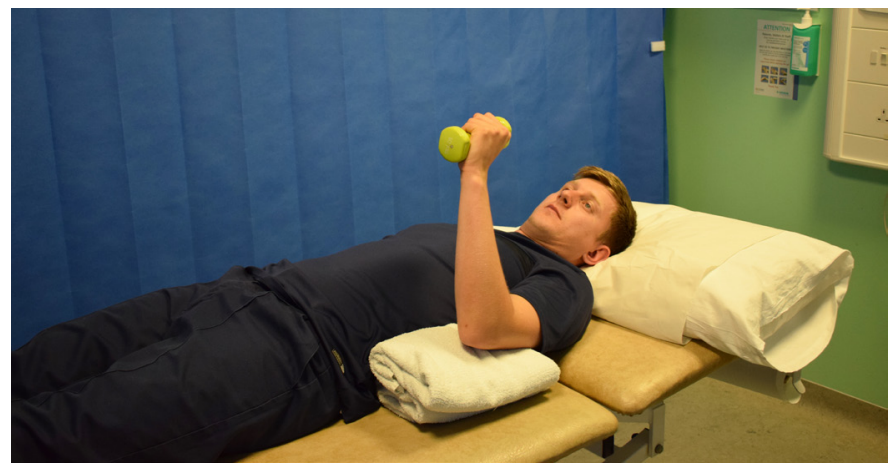

Note. Left panel: Starting position. Right panel: End position.

\section{Figure 5}

Therapist-Resisted Isometric Internal Rotation from Neutral, Performed in Supine

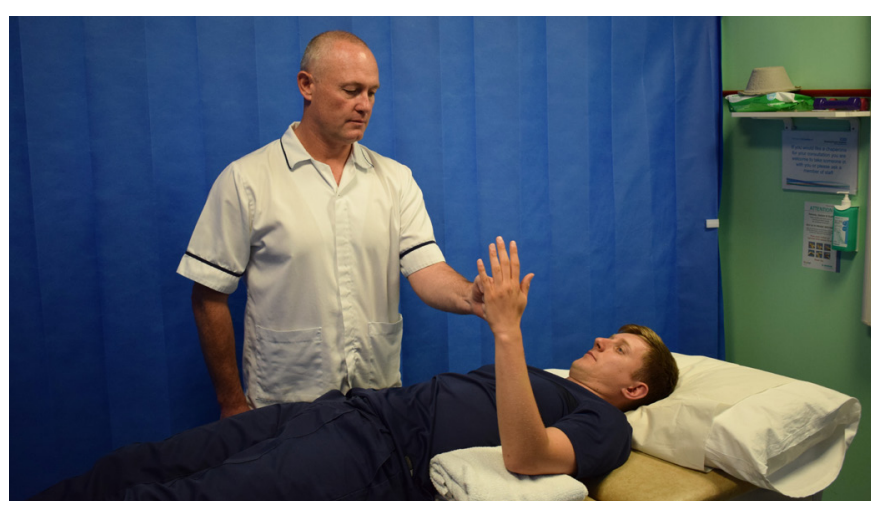

it is vital to support a judicious and progressive rehabilitation approach, rather than one determined by a time-based assumption. Any concerns with patients continuing to fail our evaluation-based tests at this stage should prompt discussion with the surgical team.

TSR with an SP or ST (12-weeks post-surgery) For patients who have undergone anatomic TSR using an SP or ST approach, a more cautionary route is taken with progression into functional reintegration. We want to allow time for sufficient healing before starting strengthening work, as suture cut through is the mode of failure that accounts for $97 \%$ of ST and SP repairs following anatomic TSR (Schrock et al, 2016). There is reasonable consensus from the rotator cuff repair literature that this should be considered from 12 weeks post-repair when there is sufficient bone tendon integration to started loaded rehabilitation (Ahmad et al., 2015; Thigpen et al., 2016). Even though the subscapularis may not have been diseased, it has nevertheless undergone surgical division and repair, so it would seem reasonable to take such a view. Patients are progressed if they pass the evaluation-based criteria tests. If they fail these tests, we follow the rationale discussed above for the LTO.

Loaded "functional reintegration" exercise progressions Rotator cuff conditioning is prescribed in a graduated manner using exercises that have shown low subscapularis activity

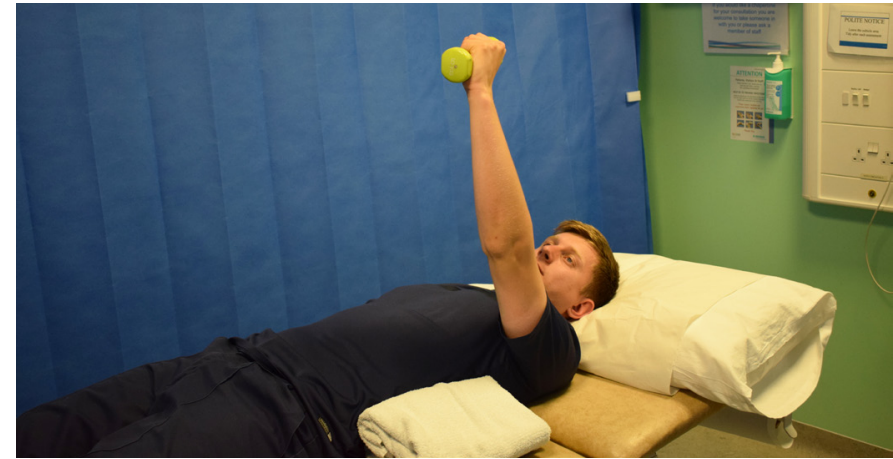

towards those where activity is higher (Jung et al., 2016; Thigpen et al., 2016), thereby fostering an incremental and controlled challenge for the healing tissues. We use hand weights or resistance bands with low load and high repetition exercises to promote stamina and endurance to replicate the patient's functional work physiology (Fisher et al., 2017). These are commonly employed due to their convenience and ease of application with a supportive goal-setting approach, which helps with exercise adherence, an important facet of a successful rehabilitation outcome (Picha \& Howell, 2018).

Loaded external rotation, either supine or standing, have shown low subscapularis activity whilst strongly recruiting the external rotators (Edwards et al., 2017; Thigpen et al., 2016), as also shown with forward flexion-type exercise progressions (Wattanaprakornkul et al., 2011). When tolerated well, these practical and functional exercises can be progressed to incorporate internal rotation and extension-type exercises that have shown increasing subscapularis activity (Edwards et al., 2017; Wattanaprakornkul et al., 2011).

Once patients are progressing with their rotator cuff conditioning, exercises that target the deltoid and scapular complex can be included, such as scapular plane flexion to $90^{\circ}$ then $120^{\circ}$, and standing rowing-type exercises (Castelein et al., 2016; Thigpen et al., 2016). The aim of these exercise progressions should always be driven by the patient's functional demands, which vary, making a bespoke approach preferable to a rigid framework. It is also important to ensure the resistance exercises incorporate education of normal movement patterns as well as the motor control work that was started in the active recovery phase.

\section{Functional rehabilitation expectations}

Rehabilitation continues until the patent's aims and goals have been achieved, as discussed in the pre-operative clinic and during rehabilitation. Whilst it can be difficult to predict the functional outcome for any given patient, there is a growing body of quantitative research that can help us provide patients with some practical guidance (Table 1) and help set realistic expectations, fostering a collaborative and holistic rehabilitation approach following TSR for OA. Furthermore, recent work has shown patients' functional improvements at 6 months are 


\section{Table 1}

Functional Outcomes Following Total Shoulder Replacement

\begin{tabular}{|c|c|c|c|c|c|}
\hline \multirow[t]{2}{*}{ Author (year) } & \multicolumn{5}{|c|}{ Post-operative measurements } \\
\hline & Pain & Flexion $\left(^{\circ}\right)$ & $\begin{array}{l}\text { External rotation in } \\
\text { adduction }\left(^{\circ}\right)\end{array}$ & $\begin{array}{l}\text { Internal } \\
\text { rotation }\left(^{\circ}\right)\end{array}$ & Notes \\
\hline Young et al. (2011) & $11.1^{\mathrm{a}}$ & 125 & 30 & & 10-year follow-up \\
\hline Razmjou et al. (2014) & & 131 & 47 & & 2-year follow-up \\
\hline \multirow[t]{2}{*}{$\begin{array}{l}\text { Dernard and Lädermann } \\
\text { (2016) }\end{array}$} & $0.7^{b}$ & 142 & 62 & L3 & $\begin{array}{l}\text { Immediate ROM group at } \\
\text { 1-year follow-up }\end{array}$ \\
\hline & $1.0^{b}$ & 146 & 57 & L1 & $\begin{array}{l}\text { Delayed ROM group at 1-year } \\
\text { follow-up }\end{array}$ \\
\hline
\end{tabular}

Note. $\mathrm{ROM}=$ range of movement.

a Pain score where $0=$ intolerable pain and $15=$ no pain. ${ }^{b}$ Visual Analogue Scale where $0=$ no pain.

maintained through 15 years postoperatively, meaning patients can be confident in the longevity of their functional recovery and independence (Raiss et al., 2014).

Some patients will also be keen to return to their leisure activities following TSR, and rehabilitation should be tailored to meet these demands. A recent meta-analysis has found that $92.6 \%$ of patients undergoing anatomic TSR return to sport (Liu et al., 2018). The most common sporting activities reported were swimming, golf, fitness sports (defined as lightweight training and/or gym attendance of more than 2 hours per week) and tennis. It should be noted, however, that the ramifications of sport participation on implant survivorship and/or complications are not fully understood at present. This is particularly important to reconcile when faced with a younger or more active patient, who may want to rehabilitate back to a number of sports or hobbies (Sowa et al., 2017).

\section{CONCLUSION}

Rehabilitation following anatomic TSR continues to advance with an evolving evidence base helping to inform our decisionmaking approach and patient care. There is a lack of evidence that shows one rehabilitation approach to be more efficacious than another. Therefore, rehabilitation protocols are often based on the available, current scientific understanding along with the experience of those clinicians that regularly manage such patient cohorts

We hope that our approach, which utilises where possible the current scientific evidence, a collaborative multidisciplinary approach, and evaluation-based criteria, provides a guideline within which to optimally rehabilitate anatomic TSR patients. Future work should aim to inform this methodology with clinical outcomes to validate the rehabilitation approach and develop further thinking in this area. Physiotherapists should be open to the exciting developments that can be utilised to improve care, such as medical ultrasound, where real-time imaging could be used to help assess the healing tissues and drive rehabilitation decision-making processes. The growing understanding of qualitative factors, such as the psychosocial, also offer potential to develop strategies and methods to improve patient experience and outcomes.

\section{KEY POINTS}

1. A collaborative evidenced and evaluation-based approach coupled with a thorough understanding of the surgical technique and factors that can lead to a poor clinical result are vital for optimising patient outcomes following anatomic TSR.

2. This clinical commentary presents a new evaluation-based rehabilitation approach to optimise the patient outcome following anatomic TSR.

\section{DISCLOSURES}

No funding was received for this research. There are no conflicts of interest which may be perceived to interfere with or bias this study.

\section{PERMISSIONS}

Permission was obtained for the reproduction of the photographs included in this article.

\section{ADDRESS FOR CORRESPONDENCE}

James Blacknall, Advanced practice shoulder and elbow physiotherapist, Trauma and Orthopaedics Department, Sherwood Forest Hospitals NHS Foundation Trust, Mansfield Road, Sutton-in-Ashfield, Nottinghamshire, NG17 4JL, United Kingdom.

Email: j.blacknall@nhs.net

\section{REFERENCES}

Ahmad, S., Haber, M., \& Bokor, D. J. (2015). The influence of intraoperative factors and postoperative rehabilitation compliance on the integrity of the rotator cuff after arthroscopic repair. Journal of Shoulder and Elbow Surgery, 24(2), 229-235. https://doi.org/10.1016/j.jse.2014.06.050

Alta, T. D., de Toledo, J. M., Veeger, H. E. J, Janssen, T. W., \& Willems, W. J. (2014). The active and passive kinematic difference between primary reverse and total shoulder protheses. Journal of Shoulder and Elbow Surgery, 23(9), 1395-1402. https://doi.org/10.1016/j.jse.2014.01.040 
Armstrong, A. D., Southam, J. D., Horne, A. H., Hollenbeak, C. S., Flemming, D. J., \& Kothari, M. J. (2016). Subscapularis function after total shoulder arthroplasty: Electromyography, ultrasound, and clinical correlation. Journal of Shoulder and Elbow Surgery, 25(10), 1674-1680. https://doi. org/10.1016/j.jse.2016.02.018

Bandura, A. (1977). Self-efficacy: Toward a unifying theory of behavioral change. Psychological Review, 84(2), 191-215. https://doi. org/10.1037/0033-295X.84.2.191

Blacknall, J., \& Neumann, L. (2011). Rehabilitation following reverse total shoulder replacement. Shoulder \& Elbow, 3(4), 232-240. https://doi org/10.1111/j.1758-5740.2011.00138.x

Bohsali, K. I., Bois, A. J., \& Wirth, M. A. (2017). Complications of shoulder arthroplasty. Journal of Bone and Joint Surgery (American), 99(3), 256269. https://doi.org/10.2106/JBJS.16.00935

Browne, A. O., Hoffmeyer, P., Tanaka, S., An, K. N., \& Morrey, B. F. (1990). Glenohumeral elevation studied in three dimensions. Journal of Bone and Joint Surgery (British), 72(5), 843-845.

Bullock, G. S., Garrigues, G. E., Ledbetter, L., \& Kennedy, J. (2019). A systematic review of proposed rehabilitation guidelines following anatomic and reverse shoulder arthroplasty. Journal of Orthopaedic \& Sports Physical Therapy, 49(5), 337-346. https://doi.org/10.2519/jospt.2019.8616

Castelein, B., Cagnie, B., Parlevliet, T., \& Cools, A. (2016). Superficial and deep scapulothoracic muscle electromyographic activity during elevation exercises in the scapular plane. Journal of Orthopaedic \& Sports Physical Therapy, 46(3), 184-193. https://doi.org/10.2519/jospt.2016.5927

Chester, R., Jerosch-Herold, C., Lewis, J. \& Shepstone, L. (2018). Psychological factors are associated with the outcome of physiotherapy for people with shoulder pain: A multicentre longitudinal cohort study. British Journal of Sports Medicine, 52(4), 269-275. https://doi.org/10.1136/ bjsports-2016-096084

Cho, C. H., Song, K. S., Hwang, I., Coats-Thomas, M. S., \& Warner J. J. P. (2017). Changes in psychological status and health-related quality of life following total shoulder arthroplasty. Journal of Bone and Joint Surgery (American), 99(12), 1030-1035. https://doi.org/10.2106/JBJS.16.00954

Choate, S. W., Kwapisz, A., Momaya, A. M., Hawkins, R. J., \& Tokish, J. M. (2018). Outcome for subscapularis management techniques in shoulder arthroplasty: a systematic review. Journal of Shoulder and Elbow Surgery, 27(2), 363-370. https://doi.org/10.1016/j.jse.2017.08.003

Denard, P., \& Lädermann, A. (2016). Immediate versus delayed passive range of motion following total shoulder arthroplasty. Journal of Shoulder and Elbow Surgery, 25(12), 1918-1924. https://doi.org/10.1016/j. jse.2016.07.032

de Toledo, J. M., Loss, J. F., Janssen, T. W., van der Scheer, J. W., Alta, T. D., Willems, W. J., \& Veeger, H. E. J. (2012). Kinematic evaluation of patients with total and reverse shoulder arthroplasty during rehabilitation exercises with different loads. Clinical Biomechanics, 27(8), 793-800. https://doi. org/10.1016/j.clinbiomech.2012.04.009

Edwards, P. K., Ebert, J. R., Littlewood, C., Ackland, T., \& Wang, A. (2017). A systematic review of electromyography studies in normal shoulders to inform postoperative rehabilitation following rotator cuff repair. Journal of Orthopaedic \& Sports Physical Therapy, 47(12), 931-944. https://doi. org/10.2519/jospt.2017.7271

Farng, E., Zingmond, D., Krenek, L., SooHoo, N. F. (2011). Factors predicting complication rates after primary shoulder arthroplasty. Journal of Shoulder and Elbow Surgery, 20(4), 557-563. https://doi.org/10.1016/j. jse.2010.11.005

Fisher, J., Steele, J., \& Smith, D. (2017). High- and low-load resistance training: Interpretation and practical application of current research findings. Sports Medicine, 47(3), 393-400. https://doi.org/10.1007/ s40279-016-0602-1

Gil, J. A., Goodman, A. D., \& Mulcahey, M. K. (2018). Psychological factors affecting outcomes after elective shoulder surgery. Journal of the American Academy of Orthopaedic Surgeons, 26(5), e98-e104. https:// doi.org/10.5435/JAAOS-D-16-00827
Gurney, A. B., Mermier, C., LaPlante, M., Majumdar, A., O'Neill, K., Shewman, T., \& Gurney, J. S. (2016). Shoulder electromyography measurements during activities of daily living and routine rehabilitation exercises. Journal of Orthopaedic \& Sports Physical Therapy, 46(5), 375383. https://doi.org/10.2519/jospt.2016.6090

Henn, F. R., Ghomrawi, H., Rutledge, J. R., Mazumdar, M., Mancuso, C. A., \& Marx, R. G. (2011). Preoperative patient expectations of total shoulder arthroplasty. Journal of Bone and Joint Surgery (American), 93(22), 2110-2115.

Jung, M. C., Kim, S. J., Rhee, J. J., \& Lee, D. H. (2016). Electromyographic activities of the subscapularis, supraspinatus and infraspinatus muscles during passive shoulder and active elbow exercises. Knee Surgery, Sports Traumatology, Arthroscopy, 24, 2238-2243. https://doi.org/10.1007/ s00167-015-3586-8

Liu, J. N., Steinhaus, M. E., Garcia, G. H., Chang, B., Fields, K., Dines, D. M., Warren, R. F., \& Gulotta, L. V. (2018). Return to sport after shoulder arthroplasty: A systematic review and meta-analysis. Knee Surgery, Sports Traumatology, Arthroscopy, 26, 100-112. https://doi.org/10.1007/s00167017-4547-1

Malhas, A., Rashid, A., Copas, D., Bale, S., \& Trail, I. (2016). Glenoid bone loss in primary and revision shoulder arthroplasty. Shoulder \& Elbow, 8(4), 229-240. https://doi.org/10.1177/1758573216648601

Matsen, F. A., Rockwood, C. A., Wirth, M. A., Lippitt, S. B., \& Parsons, M. (2004). Glenohumeral arthritis and its management. In C. A. Rockwood, F. A. Matsen, M. A. Wirth \& S. B. Lippitt (Eds.), The Shoulder (3rd ed., pp. 879-1007). Saunders.

Mazuquin, B. F., Wright, A. C., Russell, S., Monga, P., Selfe, J., \& Richards, J. (2018). Effectiveness of early compared with conservative rehabilitation for patients having rotator cuff repair surgery: An overview of systematic reviews. British Journal of Sports Medicine, 52(2), 111-121. https://doi. org/10.1136/bjsports-2016-095963

Mueller, M., \& Hoy, G. (2014). Soft tissue balancing in total shoulder replacement. Current Reviews in Musculoskeletal Medicine, 7, 16-21. https://doi.org/10.1007/s12178-013-9195-6

Picha, K. J., \& Howell, D. M. (2018). A model to increase rehabilitation adherence to home exercise programmes in patients with varying levels of self-efficacy. Musculoskeletal Care, 16(1), 233-237. https://doi. org/10.1002/msc. 1194

Raiss, P., Bruckner, T., Rickert, M., \& Walch, G. (2014). Longitudinal observational study of total shoulder replacements with cement: Fifteen to twenty-year follow-up. Journal of Bone and Joint Surgery (American), 96(30, 198-205. https://doi.org/10.2106/jbjs.m.00079

Razmjou, H., Stratford, P., Kennedy, D., \& Holtby, R. (2014). Pattern of recovery following total shoulder arthroplasty and humeral head replacement. BMC Musculoskeletal Disorders, 15, 306. https://doi. org/10.1186/1471-2474-15-306

Schrock, J. B., Kraeutler, M. J., Houck, D. A., Provenzano, G. G., McCarty, E. C., \& Bravman, J. T. (2016). Lesser tuberosity osteotomy and subscapularis tenotomy repair techniques during total shoulder arthroplasty: A metaanalysis of cadaveric studies. Clinical Biomechanics, 40, 33-36. https://doi. org/10.1016/j.clinbiomech.2016.10.013

Shi, L. L., Jiang, J. J., Ek, E. T., \& Higgins, L. D. (2015). Failure of the lesser tuberosity osteotomy after total shoulder arthroplasty. Journal of Shoulder and Elbow Surgery, 24(2), 203-209. https://doi.org/10.1016/j. jse.2014.05.006

Singh, J. A., Sperling, J. W., \& Cofield, R. H. (2011) Revision surgery following total shoulder arthroplasty: Analysis of 2588 shoulders over three decades 1976 to 2008). Journal of Bone and Joint Surgery (British), 93(11), 15131517. https://doi.org/10.1302/0301-620X.93B11.26938

Sowa, B., Bochenek, M., Bülhoff, M., Zeifang, F., Loew, M., Bruckner, T., \& Raiss, P. (2017). The medium- and long-term outcome of total shoulder arthroplasty for primary glenohumeral osteoarthritis in middleaged patients. Bone and Joint Journal, 99-B(7), 939-943. https://doi. org/10.1302/0301-620X.99B7.BJJ-2016-1365.R1 
Stephens, S. P., Spencer, E. E., \& Wirth, M. A. (2017). Radiographic results of augmented all-polyethylene glenoids in the presence of posterior glenoid bone loss during total shoulder arthroplasty. Journal of Shoulder and Elbow Surgery, 26(5), 798-803. https://doi.org/10.1016/j.jse.2016.09.053

Styron, J. F., Higuera, C. A., Strnad, G \& lannotti, J. P. (2015). Greater patient confidence yields greater functional outcomes after primary total shoulder arthroplasty. Journal of Shoulder and Elbow Surgery, 24(8), 1263-1267. https://doi.org/10.1016/j.jse.2015.04.018

Thigpen, C. A., Shaffer, M. A., Gaunt, B. W., Leggin, B. G., Williams, G. R., \& Wilcox, R. B. (2016). The American Society of Shoulder and Elbow Therapists' consensus statement on rehabilitation following arthroscopic rotator cuff repair. Journal of Shoulder and Elbow Surgery, 25(4), 521535. https://doi.org/10.1016/j.jse.2015.12.018

Tokish, J. M., Kissenberth, M. J., Tolan, S. J., Salim, T. I., Tadlock, J., Kellam, T., Long, C. D., Crawford, A., Lonergan, K. T., Hawkins, R. J., \& Shanley, E. (2017). Resilience correlates with outcomes after total shoulder arthroplasty. Journal of Shoulder and Elbow Surgery, 26(5), 752-756. https://doi.org/10.1016/j.jse.2016.12.070
Wattanaprakornkul, D., Cathers, I., Halaki, M., Ginn, K. A. (2011). The rotator cuff muscles have a direction specific recruitment pattern during shoulder flexion and extension exercises. Journal of Science and Medicine in Sport, 14(5), 376-382. https://doi.org/10.1016/j.jsams.2011.01.001

Werner, B. C., Wong, A. C., Chang, B., Craig, E. V., Dines, D. M., Warren, R. F \& Gulotta, L. V. (2017). Depression and patient-reported outcomes following total shoulder arthroplasty. Journal of Bone and Joint Surgery (American), 99(8), 688-695. https://doi.org/10.2106/JBJS.16.00541

Wolff, A. L., \& Rosenzweig, L. (2017). Anatomical and biomechanical framework for shoulder arthroplasty rehabilitation. Journal of Hand Therapy, 30(2), 167-174. https://doi.org/10.1016/j.jht.2017.05.009

Young, A., Walch, G., Boileau, P., Favard, L., Gohlke, F., Lowe, M., \& Mole D. (2011). A multicentre study of the long-term results of using a flat-back polyethylene glenoid component in shoulder replacement for primary osteoarthritis. Journal of Bone and Joint Surgery (British), 93(2), 210-216. https://doi.org/10.1302/0301-620X.93B2.25086 


\section{Appendix A}

\section{TOTAL SHOULDER REPLACEMENT REHABILITATION PROTOCOL}

Pre-operative clinic evaluation

Patient educated regarding rehabilitation plan and any functional needs evaluated

Early phase (inpatient care onwards): "Protected mobility"

Goals

- Pain controlled

- Competent with rehabilitation programme and care of upper limb

- Independent for discharge (with or without care/support as required)

Precautions

- Check operation note/surgical team communication to clarify plan and surgery details

- Check x-rays cleared as necessary

- Sling requirement for protection and support (3-4 weeks depending on surgical approach)

- No loading of upper limb

Days $1-21$

- Patient educated regarding upper limb functional use (sleep, resting positions and simple activities of daily living)

- Patient educated regarding sling management (can be removed for exercises and simple activities of daily living, as educated above)

- Patient taught routine AAROM exercises (avoiding impingement positions): Shoulder rolls, pendulum, pulley from sitting, supine flexion, supine external rotation, standing extension

- Outpatient physiotherapy arrangements made on discharge from hospital and care continued

Intermediate phase: "Active recovery"

Goals

- Complete criterion-based evaluation for progression. Consider time from surgery, patient tissue quality, surgical considerations, patient progress with rehabilitation to date and complete clinical tests (supine active internal/external rotation, and supine active forward punch)

Precautions

- Avoid loading the upper limb to protect healing soft tissues

LTO approach at 3 weeks post-surgery

- Start weaning off sling

- Start AAROM hand-behind-back movements

- Start active motor control ROM exercises

SP/ST approach at 4 weeks post-surgery

- Start weaning off sling

- Start AAROM hand- behind-back movements

SP/ST approach at 6 weeks post-surgery

- Start active motor control ROM exercises 
Late postoperative phase: "Functional reintegration"

Goals

- Complete criterion-based evaluation for progression: Consider time from surgery, patient tissue quality, surgical considerations, patient progress with rehabilitation to date, and complete clinical tests (supine resisted internal rotation and supine loaded-forward punch)

Precautions

- Avoid large functional loads through upper limb

LTO approach 6 weeks post-surgery and SP/ST approach from 12 weeks post-surgery

- Continue AAROM and AROM motor control exercises from early and intermediate phases

- Start rotator cuff exercise work up

Supine/standing external rotation with resistance

Supine/standing forward punch with resistance

Supine/standing internal rotation with resistance

Standing extension with resistance

- Start periscapular and deltoid work up

Lateral raise in scaption $0-90^{\circ} \rightarrow 120^{\circ}$

Rowing-type exercises with resistance

Anterior deltoid progressions supine to upright sitting

LTO and SP/ST up to 24 weeks post-surgery

- Goals

Good AAROM, AROM, and strength and stability

Rehabilitation progressions toward patient's functional demands and hobbies

- Exercise work up to match patient functional demands

Problem solve or make adjustments for sports/leisure aspirations collaboratively as necessary

Discuss life-long functional adaptations and upper limb demand

Note. AROM = active range of movement; $A A R O M=$ active-assisted range of movement; $L T O=$ lesser tuberosity osteotomy; ROM = range of movement; SP = subscapularis peel; ST = subscapularis tenotomy. 